\title{
Non-Existence of Trouser-Worlds
}

\author{
Wolfgang Kundt \\ I. Institut für Theoretische Physik der Universität Hamburg
}

Received September 15, 1966

\begin{abstract}
Simple restrictions are derived on the global structure of normal hyperbolic Riemannian spaces. As a consequence, cosmological models of the trouser type are ruled out irrespective of field equations.
\end{abstract}

A well-known phenomenon in astronomy is the appearance of new visible objects in the sky. This led JORDAN to the conjecture that gravity theory might offer cosmological models in which separate spaces unite at some finite time, yielding a discrete growth of the observable matter in the universe. In order to have an imaginable model in mind, think of a 2-dimensional pair of trousers in Minkowskian 3-space such that the vertical direction is timelike: horizontal sections of this "trouser world" are two circles near the bottom, and one circle at hip level. In other words: space sections are disconnected at some time, and connected at another. However, closer inspection shows that this model does not carry a regular metric: near the saddle point its signature changes from normal hyperbolic to (positive) definite. Is this failure unavoidable, i.e. independent of imbedding, and dimension? We are going to prove a simple lemma which indeed denies the existence of trouser worlds. (Further relevant global properties will be presented by SEIFERT in a later article.)

Lemma. Consider a g-complete [1] Riemannian space of continuity class $C_{2}$ containing a continuous surface $\Sigma$, and a continuous unit vector field $u^{a}$ over $\Sigma$. Follow the geodesics which start on $\Sigma$ with direction $u^{a}$ up to (eigen) distance $s$ (from $\Sigma$ ). The "geodesic map" $\Sigma \rightarrow \Sigma(s)$ of $\Sigma$ onto the points $\Sigma(s)$ thus obtained is continuous for every $s$.

Proof. It suffices to show that the image points $x_{k}(s)$ of a converging point sequence $x_{k}$ in $\Sigma$ converge towards the image point $x(s)$ of the limit $x$ of $x_{k}$ (with respect to the coordinate topology). Note that the geodesics of a $C_{2}$-space cannot split because their differential equation satisfies a Lipschitz condition. Suppose now that for a certain $s, x_{k}(s)$ does not converge towards $x(s)$, and call $s_{0}$ the infimum of all such $s$. Consequently, the geodetic segments $g_{k}$ converge pointwise towards $g$ for all $s$ smaller than $s_{0}$; and so do their tangent vectors 
$\dot{x}^{a}:=d x^{a} / d s$, and coordinate acceleration vectors $\ddot{x}^{a}:=-\Gamma_{b c}^{a} \dot{x}^{b} \dot{x}^{c}$. For if not, one could find a subsequence whose $\dot{x}_{k}^{a}\left(s_{1}\right)$ converge towards a vector differing from $\dot{x}^{a}\left(s_{1}\right)$; which conflicts with the convergence of $g_{k}$ around $s_{1}$. Next observe that $x_{k}\left(s_{0}\right)$ converges towards $x\left(s_{0}\right)$. In order to see this we introduce a normal coordinate system $S$ in $x\left(s_{0}\right)$, and assume that convergence is violated. It is then possible to choose a subsequence of $g_{k}$ whose intersections with a small sphere around $x\left(s_{0}\right)$ converge towards a point not contained in $g$. But this contradicts the fact that $\dot{x}^{a}(s)$ converges for all $s<s_{0}$, and that $\ddot{x}^{a}$ is a first order quantity at the origin of $S$.

Finally we see that $x_{k}(s)$ converges towards $x(s)$ for an open interval of distances $s$ containing $s_{0}$. This follows from the continuous dependence of solutions of differential equations on their initial values in sufficiently small domains: $x_{k}(s) \rightarrow x(s)$ holds for $x_{k}\left(s_{0}\right) \rightarrow x\left(s_{0}\right), \dot{x}_{k}^{a}\left(s_{0}\right) \rightarrow \dot{x}^{a}\left(s_{0}\right)$. The existence of an $s_{0}$ has thereby been refuted.

The lemma has interesting consequences. Recall for instance that the continuous image of a connected (compact) manifold is connected (compact), and that a simply connected image implies a simply connected inverse image. If in cosmology, $\Sigma$ is taken to be a spacelike hypersurface intersecting every matter worldline, and if the matter 4 -velocity is chosen as $u^{a}$, then the lemma implies that matter can never separate into disjoint pieces.

In conclusion, let us define a trouser world as a spacetime model which a) can be divided, by means of two space sections $\Sigma_{ \pm}$, into three disjoint parts: the future of $\Sigma_{+}$, the past of $\Sigma_{-}$, and the rest, and such that b) $\Sigma_{+}$is connected whereas $\Sigma_{-}$consists of at least two connectivity components $\Sigma_{j}$ with disjoint past, c) timelike geodesics meeting $\Sigma_{+}$meet $\Sigma_{-}$within a uniform interval of eigen time, and d) for each $\Sigma_{j}$ there exists a timelike geodesic meeting both $\Sigma_{j}$, and $\Sigma_{+}$. This definition expresses what we understand by "uniting spaces". The lemma implies that such a world cannot exist: Choose $\Sigma_{+}$as the surface $\Sigma$ from the lemma, and take for $u^{a}$ a past directed timelike vector field such that in some points $x_{j}$ of $\Sigma_{+}, u^{a}$ agrees with the tangent of a geodesic meeting $\Sigma_{j}$. For sufficiently large $s$, the geodesic image $\Sigma(s)$ must have swept past $\Sigma_{-}$, i.e. must have torn if the past of $\Sigma_{-}$was disconnected; which contradicts the lemma.

Acknowledgements. These problems have been stimulated by WOLFGANG BUDICH, and developed in discussions with JoHn Madore, Professor A. Papapetrou, and HANS-JÜRGEN SEIFERT.

\section{Literatur}

[1] Kundt, W.: Z. Physik 172, 488 (1963). 